\title{
Desenvolvimento e validação de um protótipo para a coleta de sinais vitais
}

\author{
Jorge Arthur Schneider Aranda, Marta Rosecler Bez, Juliano Varella de Carvalho \\ Grupo de Computação Aplicada - Universidade FEEVALE \\ ERS 239, 2755 - Vila Nova, Novo Hamburgo - RS, 93525-075 \\ jorgearthurs@hotmail.com, julianovc@feevale.br, martarb@feevale.br
}

\begin{abstract}
This paper presents the development of a prototype for the monitoring of vital signs through Arduino and e-health prototyping boards. The methodology used was exploratory and the prototype was validated with athletes of an amateur volleyball team and its coach. After the use was applied a satisfaction questionnaire to the athletes and coach. In both cases the receptivity to use this type of technology was very good.

Resumo. Este artigo apresenta o desenvolvimento de um protótipo para o monitoramento de sinais vitais através das placas de prototipagem Arduino e e-Health. A metodologia utilizada foi exploratória e o protótipo foi validado com atletas de um time amador de vôlei feminino e seu treinador. Após o uso foi aplicado um questionário de satisfação às atletas e ao treinador. Em ambos os casos, os participantes demonstraram receptividade para o uso deste tipo de tecnologia.
\end{abstract}

\section{Introdução}

A otimização da carga de treinamento de atletas deve levar em consideração suas características pessoais. Segundo Dücking et al. (2016), atletas em geral necessitam reajustar seus treinamentos diariamente a fim de otimizar seu desempenho, evitando a fadiga, o treinamento demasiado e outros efeitos indesejáveis para a sua saúde.

Atualmente existem soluções que permitem a coleta de sinais vitais, tais como plataformas de prototipagem eletrônica (Arduino, Raspbery, etc). A partir de uma investigação prévia realizada nesta pesquisa, encontrou-se também a placa e-Health, especializada na coleta de sinais vitais de um usuário.

Um exemplo de uso de tecnologias similares ao da placa supracitada, é o recurso de telemedicina. Este recurso permite que os pacientes se conectem a provedores via telefone ou uma webcam para discutir suas preocupações médicas e receber orientação sobre o que devem fazer (CARVALHO, 2015), incluindo ir a uma farmácia para pegar uma prescrição ou verificar se os sintomas não pioraram antes de ver um médico em pessoa para receber o tratamento adequado.

Existem exemplos de serviços tal qual a plataforma de telemedicina da Unitcare Saúde. A solução oferece sete equipamentos modulares que podem monitorar doenças de alta complexidade através de uma Central de Exames remota (TIZATTO, s.d.). 
Este serviço se baseia em oferecer aparelhos de captura de sinais vitais, tais como pressão sanguínea, oximetria, temperatura, peso, índice glicêmico, que são conectados via bluetooth até um receptor, que transmite as informações para uma central onde os dados serão analisados e armazenados, através de um sinal de internet ou rede celular, a exemplo de um celular comum. Dessa forma, o paciente é monitorado 24 horas por dia, sete dias por semana, sem interrupções. Sofrendo atualizações a cada 30 segundos, os dados podem ser acessados pelos profissionais de saúde autorizados de qualquer computador com acesso a internet, tablet ou smarthphone, em tempo real e de forma eficaz. Todos os dados são criptografados e a segurança da informação é tratada como prioridade (TIZATTO, s.d.).

Todos esses cuidados à distância demandam de tecnologias diversas para seu funcionamento. Uma das alternativas é utilizar plataformas de prototipagem, que devido a um processamento satisfatório para este tipo de aplicação e de tamanho reduzido, podem ser de grande utilidade na área de saúde.

Dentre os trabalhos relacionados, podemos citar o trabalho de Vargas Escobar e Salinas (2016). Este trabalho utiliza-se da plataforma de prototipagem Arduino para monitorar a frequência cardíaca, permitindo que em caso de uma situação mais grave o próprio dispositivo avise o hospital ou clínica, cadastrada previamente pelo paciente, para enviar o serviço de emergência com rapidez e agilidade.

Há também os estudos com a própria placa e-Health, conduzidos por Rakáy et al. (2015), onde foram analisados os sensores de batimentos cardíacos, acelerômetro, temperatura da pele e fluxo respiratório. Neste estudo a comunicação empregada foi wireless, onde os dados foram enviados para outro receptor wireless conectado a um Arduino e este, por sua vez, conectado a um computador onde eram exibidos os resultado. Ou seja, neste caso os dados não foram enviados para a nuvem (cloud).

O artigo em questão está dividido em seis seções. A primeira é esta introdução. A segunda seção aborda a metodologia empregada na pesquisa. A terceira e quarta seções abordam as tecnologias utilizadas e o desenvolvimento do protótipo. A seção cinco discute a validação realizada e, por fim, conclusões são apresentadas na sexta seção.

\section{Metodologia}

Este estudo trata-se de uma pesquisa experimental, composta de um grupo voluntário. $\mathrm{O}$ protótipo foi desenvolvido e validado com seis atletas da equipe feminina de vôlei da Universidade Feevale, em dois dias diferentes, sempre com uma coleta anterior ao treino e outra posterior, totalizando quatro coletas. Os dados foram enviados para a nuvem e puderam ser visualizados por meio de um aplicativo para celular.

A população estudada é composta de indivíduos membros de um time de vôlei amador. O tipo de amostra é não probabilística, composta por 7 indivíduos, 6 atletas dos quais foram obtidos os dados e um treinador que os analisou. Os sinais capturados foram batimentos cardíacos, temperatura e pressão arterial. Todos os participantes assinaram o termo de livre esclarecimento, sendo esta pesquisa encaminhada ao comitê de ética da Universidade, a partir de seu cadastro na Plataforma Brasil. 
A aferição foi efetuada durante dois momentos, capturando os sinais vitais dos atletas antes e depois do treino. O protótipo estava conectado na rede Wi-Fi compartilhada de um smartphone que possui conexão $3 \mathrm{G}$. Esta coleta consistia em efetuar upload do código para o Arduino, desligar o equipamento e, em seguida, conectar um dos sensores, sempre respeitando sua ordem $\left(1^{\circ}\right.$ batimentos cardíacos e $2^{\circ}$ temperatura) e, por fim, ligava-se o equipamento para de fato coletar o sinal vital.

Durante o desenvolvimento desta metodologia, foram questionados alguns atletas e o técnico do time de vôlei da universidade Feevale. Conforme este contato, delimitou-se que uma possível maneira para efetuar a coleta seria a mencionada na metodologia apresentada anteriormente.

Com base na literatura citada, questiona-se se é possível, através da placa eHealth, capturar dados de atletas que possam gerar informações de apoio a treinadores e preparadores físicos? Para responder a este questionamento, o objetivo geral deste trabalho foi o de criar um protótipo em Arduino, em conjunto com o Shield e-Health, que coletou sinais vitais dos atletas e enviou esses dados para nuvem (cloud), para o repositório de dados Thingspeak, via comunicação wireless.

\section{Tecnologias empregadas}

No decorrer deste trabalho, diversas tecnologias foram empregadas. Desde plataformas de prototipagem até repositório de dados na nuvem. As plataformas de prototipagem consistem em dispositivos de placa única, com um micro controlador (WHAT IS ARDUINO, 2015) que permitem ao usuário executar as mais diversas funções, como automação residencial, automação industrial e também aplicações voltadas para a saúde. Nesta seção serão abordados alguns conceitos importantes destes tipos de placas e, por fim, um estudo da placa Arduino, que foi utilizada para a construção do protótipo deste trabalho.

Arduino (Figura 1) é uma placa de prototipagem em uma plataforma física de computação de código aberto, baseada em uma simples placa micro controladora, e um ambiente de desenvolvimento para escrever o código para a placa (WHAT IS ARDUINO, 2015). A placa de prototipagem Arduino pode ser utilizada para a criação de objetos interativos, permitindo entradas desde sensores, que controlam uma variedade de motores, luzes ou outras saídas físicas. Projetos em Arduino podem ser independentes, ou podem comunicar-se com software rodando através de um computador. Os circuitos podem ser montados manualmente ou comprados prémontados; o software para programação é de código-livre e pode ser baixado gratuitamente (WHAT IS ARDUINO, 2015). 


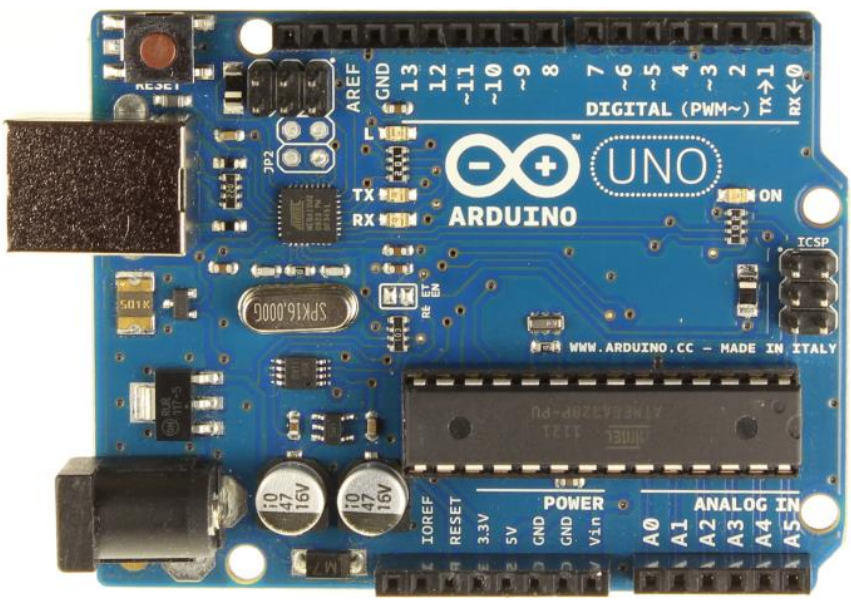

Figura 1 - Arduino (Lab de Garagem, 2015).

O Shield e-Health (Figura 2) permite, em conjunto com uma placa de prototipagem como Arduino ou Raspberry $P i$, executar aplicações biométricas e médicas onde o monitoramento de sinais vitais é necessário, usando até 9 sensores diferentes: oxigenação sanguínea (SpO2), fluxo de ar (respiração), temperatura do corpo, eletrocardiograma (ECG), glicosímetro, resposta galvânica da pele (GSR sudorese), pressão arterial (esfigmomanômetro), posição do paciente (acelerômetro) e músculo / sensor de eletromiografia (EMG). Esses sinais vitais podem ser utilizados para monitorar em tempo real o estado de um paciente ou para obter dados mais sensíveis do mesmo, a fim de serem posteriormente analisados para o diagnóstico médico (COOKING HACKS, 2013).

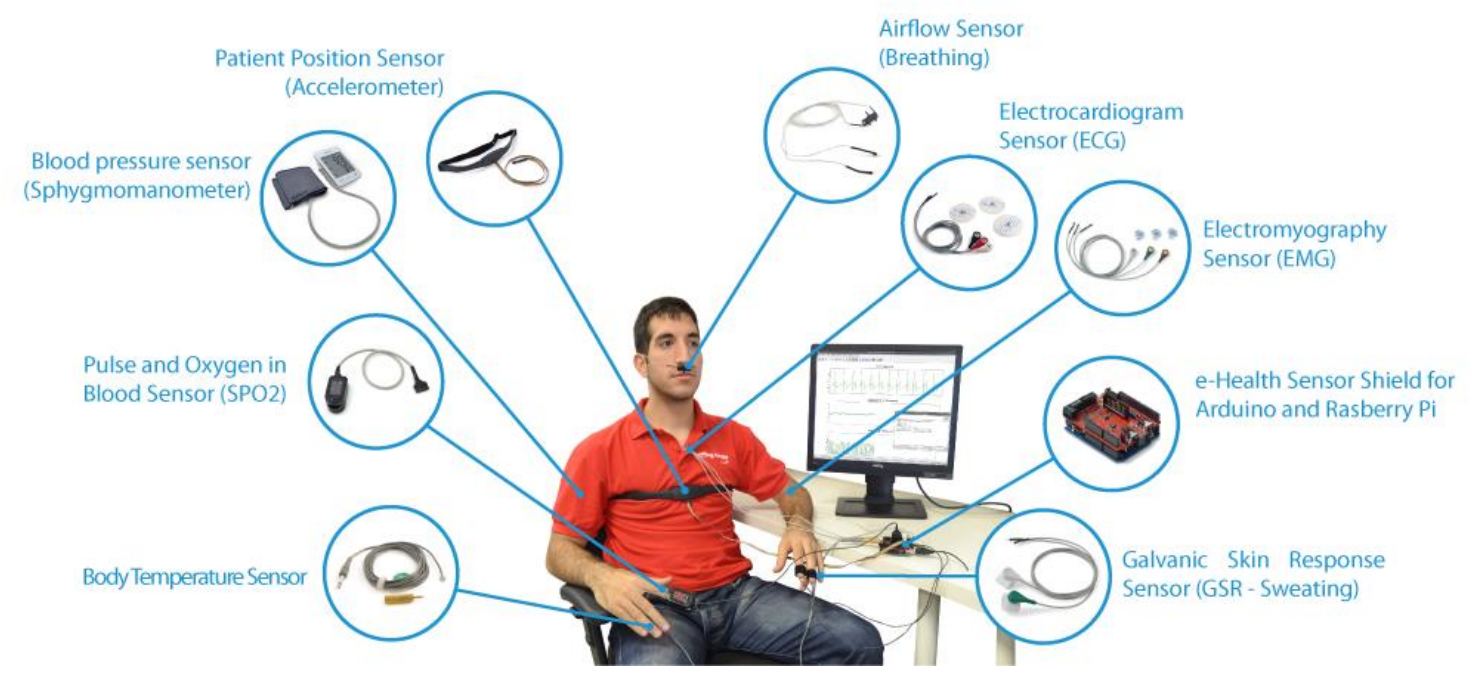

Figura 2 - Exemplo de utilização do Shield e-Health e seus diversos sensores (Cooking Hacks, 2013).

Para poder armazenar os dados coletados na nuvem (cloud), é necessário utilizar repositórios de dados (CORDEIRO, 2013), tais como o Thingspeak. Esta é uma plataforma de prestação de diversos serviços, exclusivamente direcionada para a construção de aplicações da IoT (Internet Of Things - Internet das Coisas). A plataforma 
oferece as capacidades de coleta de dados em tempo real, possibilitando que os dados recolhidos sejam exibidos na forma de gráficos, por exemplo.

\section{Desenvolvimento}

Para funcionamento da placa e-Health é necessário que a mesma esteja conectada sobre o Arduino. A placa e-Health pode receber os códigos desenvolvidos em Arduino, pois a IDE interpreta que o Arduino e a placa são o mesmo dispositivo.

A solução escolhida para enviar os dados para a nuvem foi o Shield Wi-Fi ESP8266, pois este é facilmente encontrado e adquirido, assim como o desenvolvimento para o mesmo também pode ser efetuado na IDE do Arduino. Isso permite ao protótipo ter uma conexão Wi-Fi plena, sem necessitar de códigos e recursos complexos, pois todos os protocolos necessários já estão nativos no Shield (Figura 3).

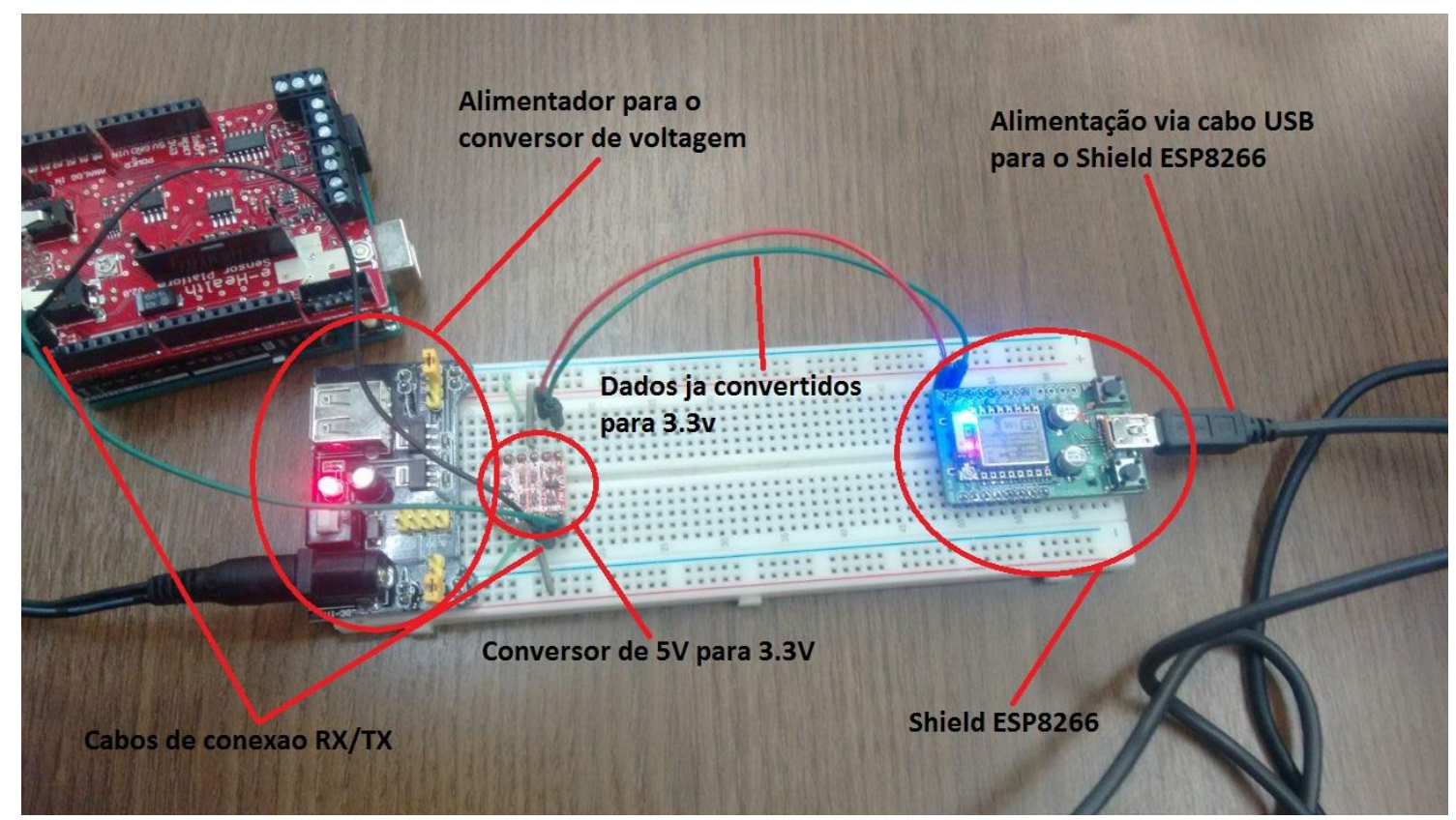

Figura 3 - Esquema de conexão do protótipo (Do autor, 2016).

Para a comunicação com o Arduino/Shield e-Health foi necessário fazer uma conexão entre os cabos RX/TX do Shield com o do protótipo, conforme demonstrado na Figura 3. Para utilizar a solução proposta também foi necessário instalar em uma protoboard um conversor de voltagem, uma vez que o Arduino envia 5v e o Shield ESP8266 apenas recebe 3.3v. Conforme apresentado na Figura 3, a placa e-Health envia os dados a partir das suas portas TX (cabo verde) e RX (cabo preto) para o conversor que, por sua vez, converte os sinais elétricos para que possam ser interpretados (evitando queimar) o Shield ESP8266.

Um aplicativo em Android foi desenvolvido para permitir a um indivíduo interessado o acompanhamento dos valores aferidos. Entende-se que para um treinador de algum time de esportes, por exemplo, é mais prático visualizar as informações em um dispositivo móvel (tablet, smartphone) do que em um computador, que nem sempre está perto dos ambientes de prática dos esportes. 
É importante reforçar que para privacidade dos atletas os nomes não foram incluídos no app. Uma vez clicado em um dos atletas, é exibida uma tela (Figura 4) onde é possível escolher o sinal vital a ser exibido. Em um primeiro momento, visualiza-se apenas o traçado gráfico efetuado pelos valores das coletas de sinais vitais. Mas, se for tocado nos pontos, são exibidas mais informações a respeito da medição, conforme Figura 4.

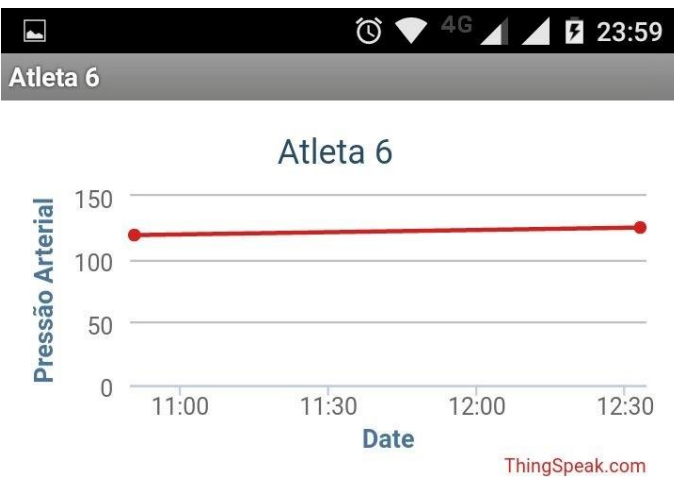

Atleta 6

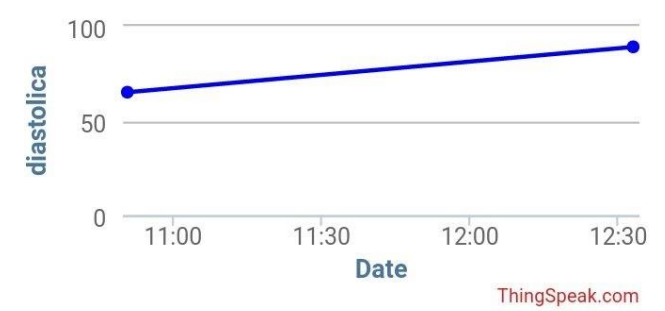

Pressão Arterial

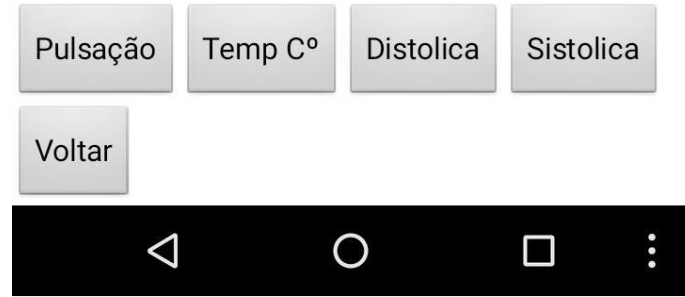

Atleta 6



Atleta 6

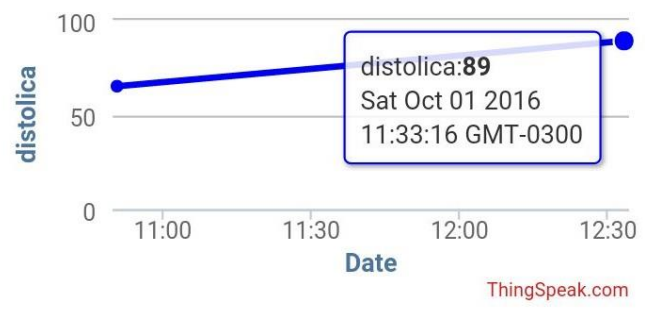

Pressão Arterial

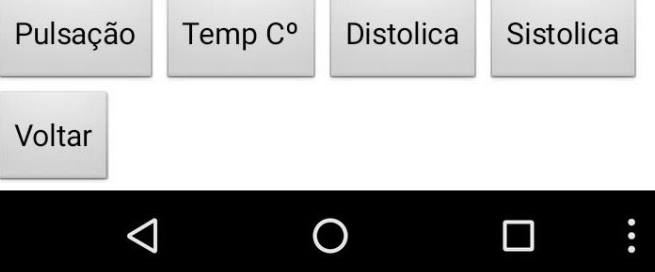

Figura 4 - Telas do aplicativo Android desenvolvido (Do autor, 2016).

Através das soluções descritas até aqui foi possível aferir os sinais vitais, enviálos através da Wi-Fi para. Por fim, armazená-los no repositório de dados Thingpseak e visualizá-los através de um smartphone.

\section{Resultados e discussão}

Uma vez concluídas as aferições, os dados foram disponibilizados para o técnico do time de vôlei analisar e, a partir destas informações, verificar se os dados observados faziam sentido de acordo com o momento pré ou pós treino. Também foram aplicados questionários com quatro atletas (duas faltaram ao treino no dia da aplicação do questionário) e com o treinador. 
Quando questionado se existiu algum tipo de desconforto ou mal estar nas medições dos sinais vitais, $100 \%$ dos respondentes afirmaram que não houve nenhum tipo de desconforto. Foi questionado se o atleta acredita que esta medição pode ajudar a melhorar o desempenho no esporte. Três dizem acreditar que sim e uma que não. Foi questionado também se a atleta ficou satisfeita com a maneira que os dados foram obtidos. Três responderam que sim e uma respondeu que não sabia ou não queria opinar.

O treinador também respondeu a um questionário composto de três perguntas. A primeira era se houve dificuldade para acessar os sinais vitais dos atletas. O treinador considerou ser fácil de acessar e ressaltou que ter essas informações no seu celular é bem mais prático que em um computador, por exemplo.

Na segunda pergunta foi questionado se esse tipo de monitoramento pode ajudar a melhorar o rendimento dos atletas. $\mathrm{O}$ treinador acredita que com os dados dos atletas sendo exibidos de forma mais ágil e prática, é possível ajustar treinos, de acordo com a fadiga do atleta, inclusive, em alguns casos, reduzindo o risco de lesões.

Por fim, foi questionado se o treinador usaria novamente esse recurso para mapear os sinais vitais dos seus atletas. $\mathrm{O}$ treinador acredita que seria interessante executar novamente estes tipos de experimentos, pois disponibiliza mais informações, lhe permitindo tomar decisões possivelmente mais acertadas.

\section{Conclusão}

Este trabalho apresentou o desenvolvimento de um protótipo, de forma a oferecer a captura de sinais vitais de maneira prática e, de certo modo, móvel. Desta forma, por exemplo, o usuário não precisaria ir seguidamente ao médico, para uma simples captura de um sinal vital específico, dependendo somente de um pequeno protótipo, do tamanho aproximado ao de um cartão de crédito, utilizando todos os recursos da placa de prototipagem Arduino.

Em conjunto com esta placa de prototipagem foi usado o Shield e-Health. Este Shield é responsável pela captura dos sinais vitais, juntamente com mais um Shield WiFi, que envia os dados para a nuvem. Dessa forma, podem ser monitoradas possíveis alterações vitais, até mesmo, permitindo a um profissional da saúde ou o próprio usuário tomar ações a partir das informações obtidas.

Através da validação efetuada juntamente da equipe de vôlei da Universidade, foi atingido o objetivo geral de construir um protótipo que permitisse coletar e enviar as informações de sinais vitais para a nuvem (cloud). Os dados foram coletados e enviados para o repositório de dados (Thingspeak) e foram acessados pelo celular do treinador da equipe.

Este trabalho teve alguns problemas não previstos no seu decorrer, o que, de certa forma, ajudou a melhorá-lo e abriu diversas novas ideias para trabalhos futuros, como, por exemplo: criar um protótipo para auxiliar idosos, como um monitor de quedas; ajustar o trabalho existente para exibir as informações aferidas em uma tela acoplada ao equipamento, permitindo a um profissional da saúde aferir populações em áreas mais isoladas, onde não há todo o equipamento tradicional. Também validar o 
quão confiáveis são os valores coletados em comparação a equipamentos já utilizados em hospitais e clínicas. Armazenar localmente os dados de sinais vitais coletados, uma vez que dados da área da saúde são bastante sensíveis, e a perda destes, por algum erro de comunicação, por exemplo, pode causar prejuízos ao usuário, uma vez que este dado não pode ser obtido naquelas mesmas condições novamente.

Se comparado aos resultados dos trabalhos relacionados, como por exemplo o trabalho de Rákay et al. (2015), que também utilizou da placa e-Health, neste trabalho foi possível enviar as informações a nuvem e permitir as mesmas serem acessadas via dispositivos móveis. No trabalho citado as coletas somente eram enviadas dentro da mesma rede Wi-fi, sem conectividade com a internet. Já em comparação com o trabalho de Vargas Escobar e Salinas (2016), este trabalho visa coletar diferentes sinais vitais, com potencial de coletar pelo menos mais 4 sinais vitais, enquanto que o trabalho mencionado coleta apenas um tipo de sinal vital(frequência cardíaca).

Por fim, entende-se que a área de eHealth é riquíssima e ainda pouco explorada, pois permite diversas possibilidades de acordo com a necessidade desejada. Basta integrá-la corretamente aos conceitos da $I o T$, permitindo resultados inovadores que podem agregar nas mais diversas áreas, seja da saúde ou esportes.

\section{Referências Bibliográficas}

Carvalho, C. (2015) " E-health: a importância e as vantagens para os mais idosos" . Disponível em: <http://agusvinnus.prodiversitas.org/revistas/Carvalho2.pdf>. Acessado em 02/06/16.

Cooking Hacks. (2013) " E-health sensor plataform v2.0 for arduino and raspberry pi" . Disponível em: <https://www.cookinghacks.com/documentation/tutorials/ehealth-biometric-sensor-platform-arduinoraspberry-pi-medical>. Acessado em 27/11/15.

Cordeiro, J. L. (2013) "The energularity” . Disponível em: <http://cesd.az/new/wpcontent/uploads/2013/05/CordeiroEnergularity2013_Paper.pdf>. Acessado em 03/11/2015.

Della Mea, V. (2001) “What is e-health (2): the death of telemedicine?” . Disponível em: <http://www.jmir.org/2001/2/e22/>. Acessado em 08/05/16.

Düking, P.; Hotho, A.; Holmberg, H.-C.; Fuss, F. K.; Sperlich, B. (2016) "Comparison of non-invasive individual monitoring of the training and health of athletes with commercially available wearable technologies". Disponível em: <https://www.ncbi.nlm.nih.gov/pmc/articles/PMC4783417/>. Acessado em 09/12/2016.

Evans, D. (2011) “A internet das coisas como a próxima evolução da internet está mudando tudo". Disponível em: <http://www.cisco.com/web/BR/assets/executives/pdf/internet_of_things_iot_ibsg_041 1 final.pdf $>$. Acessado em:= 02/05/16. 
Rákay, R.; Višňovský, M.; Galajdová, A.; Šimšík, D.(2015) “Testing Properties of Ehealth System Based on Arduino." Journal of Automation and Control, vol. 3, no. 3 (2015): 122-126. doi: 10.12691/automation-3-3-17.

Tizatto, L. s.d.. Disponível em: <http://www.unitcare.com.br/imprensa/43-unicaresaude-oferece-primeira-plataforma-de-telemedicina-para-homecare-de-altacomplexidade.> Acessado em: 08/05/16.

Vargas Escobar, L.J.; SALINAS, S.A.(2016) " e-Health prototype system for cardiac telemonitoring.". Disponível em: <https://www.ncbi.nlm.nih.gov/pubmed/28269253>. Acessado em: 02/05/2017

Wakefield, B. J.; Holman, J. E.; Ray, A.; Morse, J.; Kienzle, M. G. (2004) “ Nurse and patient communication via low-introduction and high-bandwidth home telecare systems" . Disponível em: <http://www.ncbi.nlm.nih.gov/pubmed/15165441>. Acessado em 02/06/16.

"What is arduino" (2016) Disponível em: <https://www.arduino.cc/en/Guide/Introduction>. Acessado em 09/10/2016. 